\title{
Magnetic anisotropy of polycrystalline magnetoferritin investigated by SQUID and electron magnetic resonance.
}

F. Moro, ${ }^{\mathrm{a}, 1,{ }^{*}}$ R. de Miguel, ${ }^{\mathrm{b}}$ M. Jenkins, ${ }^{\mathrm{c}}$ C. Gómez-Moreno, ${ }^{\mathrm{b}}$ D. Sells, ${ }^{\mathrm{d}}$ F. Tuna, ${ }^{\mathrm{d}}$ E. J. L. McInnes, ${ }^{\text {d,e }}$ A. Lostao, ${ }^{\text {b,f }}$ F. Luis ${ }^{c}$ and J. van Slageren ${ }^{\text {g }}$

a. School of Chemistry, University of Nottingham, NG7 2RD Nottingham, UK b. Laboratorio de Microscopías Avanzadas (LMA)_Instituto de Nanociencia de Aragón (INA), Universidad de Zaragoza, 50018 Zaragoza, Spain c. Instituto de Ciencia de Materiales de Aragón, Universidad de Zaragoza-CSIC, 50009 Zaragoza, Spain

d. EPSRC National UK EPR Facility, Photon Science Institute, University of Manchester, Manchester, M13 9PL, UK

e School of Chemistry, University of Manchester, Manchester, M13 9PL, UK

f. Fundación ARAID, Spain

g. Institut für Physikalische Chemie, Universität Stuttgart, Pfaffenwaldring 55, D70569 Stuttgart, Germany

Magnetoferritin molecules with an average inorganic core diameter of $5.7 \pm 1.6 \mathrm{~nm}$ and polycrystalline internal structure were investigated by a combination of transmission electron microscopy, magnetic susceptibility, magnetization, and electron magnetic resonance (EMR) experiments.

The temperature and frequency dependence of the magnetic susceptibility allowed for the determination of the magnetic anisotropy on an experimental time scale which spans from seconds to nanoseconds. In addition, angle-dependent EMR experiments were carried out for the determination of the nanoparticle symmetry and internal magnetic field.

Due to the large surface to volume ratio, the nanoparticles show larger and uniaxial rather than cubic anisotropies compared to bulk maghemite and magnetite.

\section{Introduction}

Ferritin and other biological nanomoulds give rise to considerable interest because they offer the possibility to synthesize size-controlled magnetic nanoparticles

\footnotetext{
* Corresponding author email: fabrizio.moro@manchester.ac.uk

1 Present address: School of Chemistry and Photon Science Institute, University of Manchester, Oxford Road, Manchester, M13 9PL, UK.
} 
(MNPs), that are rather homogeneous in shape and possess narrow size distributions.[1] These bioinspired nanomaterials enable a deeper investigation and understanding of the magnetic properties than nanoparticles obtained by other physical or chemical preparation methods. ${ }^{3}$ The protein cage of ferritin provides a confined vessel where guest species can enter and react giving a core with defined size and shape. The cavity of ferritin, i.e. apoferritin, has spherical shape with internal and external diameters of $\approx 8$ and $12 \mathrm{~nm}$, respectively. It naturally contains antiferromagnetic ferrihydrite, which, when replaced with maghemite, results in a ferrimagnetic material called magnetoferritin.[2, 3] The apoferritin thickness of around $2 \mathrm{~nm}$ is sufficient to magnetically shield the cores from each other leading to weak inter-particle magnetic interactions. Besides maghemite, many other materials have been synthesized inside apoferritin, including metal oxides [4, 5] as well as pure metals.[6-9] Furthermore, magnetoferritin is of particular interest for biomedical[10, 11] applications, such as drug delivery, hyperthermia and as contrast agents for magnetic resonance imaging (MRI).[12, 13]

The possibility of fine tuning the Fe loading in apoferritin may open the way to control the magnetic properties of magnetoferritn. A suitable chemical synthesis to this aim was reported by Wong et al.[14] where the superparamagnetic blocking temperature was found to increase approximately linearly with the iron content. Recently a more detailed analysis of the structure and magnetic properties of magnetoferritin with size from 1.6 to $6 \mathrm{~nm}$ revealed low degree of crystalline order, reduction of the magnetic moment, larger magnetic anisotropy compared to bulk materials and increased anisotropy constant with decreasing particle size. These results were explained by the contribution of surface spins located at the particle surface and at the interfaces between different crystalline domains inside each molecule.[15] These studies were carried out by static (DC) and dynamic (AC) magnetic susceptibility on an experimental time-scale from seconds to ms. However, several practical applications require to achieve a control of the magnetic susceptibility response in a wider range of time-scales. For instance, the absorption of electro-magnetic radiation by nanoparticles might drastically change under certain conditions of electro-magnetic frequency. Therefore, MNPs can turn out to be useful as good absorber for radiation detectors in a particular range of frequencies. Moreover, the application of MNPs for cancer treatment as well as microwave 
assisted magnetic recording[16] requires the understanding of their magnetic anisotropy and magnetisation dynamics behaviour as a function of radiation frequency, temperature, magnetic fields as well as direction of the particle magnetic moment with respect to the magnetic field direction. Therefore the performance of some devices can be optimised by the choice of the most suitable operational time, temperature and external magnetic field strength and direction. Electron magnetic resonance (EMR) turns out to be particularly useful for the study of the magnetic response of MNPs under microwave frequencies (nanoseconds time-scale). A temperature dependence of the resonance field $\left(\mathrm{B}_{\mathrm{r}}\right)$ and peak-to-peak linewidth $\left(\Delta_{\mathrm{B}}\right)$ of EMR spectra at X-band ( $10 \mathrm{GHz})$ of MNPs is widely reported.[17-22] However, the large broadening of the resonance linewidth makes the analysis of such spectra difficult to interpret. Frequency[22-24] and angle dependent[25-27] EMR measurements of $\mathrm{B}_{\mathrm{r}}$ and $\Delta_{\mathrm{B}}$ provide important additional information such as $\mathrm{g}$ value, damping effects, anisotropy field and symmetry which can help the interpretation of the EMR spectra. However, only few frequency and angular dependent studies have been reported so far and the interpretation is usually based on the assumption that the protein cages contain uniformly magnetised nanoparticles (single domain magnets). Herein, we report on a temperature, frequency and angular dependence study of $B_{r}$ and $\Delta_{B}$ on magnetoferritin with low degree of crystalline order due to the formation of multiple domains. To the best of our knowledge, the polycrystalline nature of the magnetoferritin core has never been considered before for the interpretation of the EMR data obtained from protein cages systems.

The paper is organised as follows. Section 2 describes in some detail the experimental techniques used in the present study. Section 3 contains the results of the morphological and magnetic characterization of the magnetoferritin sample. Section 4 is dedicated to the analysis of temperature, frequency and angular dependences of EMR data and Section 5 to the conclusions.

\section{Experimental procedure}

\subsection{Synthesis}

The synthesis of ferritin nanoparticles was carried out as previously reported [15]. A solution of horse spleen apoferritin was prepared in AMPSO (3-[(1,1-Dimethyl-2hydroxyethyl)amino]-2-hydroxy-propanesulfonic acid) $\mathrm{pH} 8.6$ at $65^{\circ} \mathrm{C}$. Aliquots of 
$\left(\mathrm{NH}_{4}\right)_{2} \mathrm{Fe}\left(\mathrm{SO}_{4}\right)_{2} \cdot \mathrm{H}_{2} \mathrm{O}$ (all reactants purchased from Sigma-Aldrich) were added every 15 minutes to the solution. Every addition of the iron salt means a theoretical load of $140 \mathrm{Fe}$ atoms per protein molecule. The reaction mixture was dialyzed against water and the dark brown magnetoferritin lyophilized.

\subsection{Transmission electron microscopy (TEM)}

A dilute solution in water was prepared from lyophilized magnetoferritin powder. A TEM holey carbon $300 \mathrm{mesh} / \mathrm{Cu}$ grid (SPI Supplies) was placed on a droplet of this solution for a minute and washed by putting it on three droplets of distilled water. The excess liquid was removed and the grid was then left drying overnight in a desiccator. When staining was required, the water-washed grid was put over three droplets of a freshly prepared $1 \%$ aurothioglucose $\left(\mathrm{C}_{6} \mathrm{H}_{11} \mathrm{AuO}_{5} \mathrm{~S} \cdot \mathrm{xH}_{2} \mathrm{O}\right.$; Sigma-Aldrich) solution and left incubating for three minutes in darkness. The samples were imaged using a Hitachi CM 30 microscope.

\subsection{Energy-dispersive $X$-ray spectroscopy (EDX)}

The ferritin nanomaterial was analyzed with a scanning electron microscope Hitachi S-3400 N equipped with a Röntec XFlash de Si(Li) EDX analyzer. A small portion of protein powder was spread on carbon. Measurements were done using variable pressure at $60 \mathrm{~Pa}$, a $10 \mathrm{~mm}$ working distance and a tension in the filament of about 15 kV. Two different micrometric areas were analyzed (SI Fig. 1 and SI Table I).

\subsection{Magnetic characterization}

The magnetic properties, magnetization and ac susceptibility, were measured in a commercial SQUID magnetometer (Quantum Design MPMS-XL) equipped with the ac measurement and the enhanced-sensitivity $\left(10^{-9} \mathrm{emu}\right)$ RSO options. The frequency $\omega / 2 \pi$ of the ac magnetic field was varied between $0.1 \mathrm{~Hz}$ and $1 \mathrm{kHz}$. The amplitude of the ac excitation field was 4 Oe in these experiments. Magnetoferritin powder samples were mixed with apiezon $\mathrm{N}$ grease to improve thermal contact and to prevent the rotation of the grains by the action of strong magnetic fields at low temperatures. The magnetic signals of the plastic sample holder and the grease were measured under 
identical conditions and then subtracted from the data. All magnetic data shown below have been normalized by the total mass of magnetoferritin.

\subsection{Electromagnetic resonance (EMR)}

L (1 GHz), S (3.86 GHz), X (9.7 GHz), K (24 GHz) and Q (34.12 GHz)-band electron magnetic resonance spectra were recorded with Bruker EMX spectrometers. W-band $(93.96 \mathrm{GHz})$ measurements were performed with Bruker Elexsys spectrometer. In all the measurements a modulation frequency of $100 \mathrm{KHz}$ and modulation amplitude of 1 $\mathrm{G}$ were used in continuous-wave and perpendicular mode. Variable temperature $\mathrm{X}$ band measurements were performed between 140 and $300 \mathrm{~K}$ by using a nitrogen cooling insert as well as down to $5 \mathrm{~K}$ by means of the He cooling insert. In order to avoid orientation effects due to the torque force induced by the EMR sweeping field, powder samples were ground and mixed with melted eicosane. Liquid suspended samples were prepared by diluting magnetoferritin in $0.4 \mathrm{M}$ glycine in phosphate buffered saline (PBS) with a freezing temperature of $200 \mathrm{~K}$. X-band angle dependent measurements were performed by mounting an EMR glass tube on a rotating support allowing rotation of the sample around the axes perpendicular to the magnetic field direction with accuracy $\pm 0.1^{\circ}$. The recorded EMR signals did not show any sign of saturation up to $100 \mathrm{~mW}$ for the entire temperature range investigated.

\section{Morphological and magnetic characterization}

\subsection{TEM analysis}

The images of the stained samples show that the iron oxide cores had grown successfully inside the inner cavity of the apoferritin molecules. Figure 1 shows typical TEM images taken on magnetoferritin samples. The black dots correspond to the iron oxide cores. Aurothioglucose binds to the carbon layer in the grid staining the metallic cores, while the protein moiety remains unmodified (Fig. 1b); the dark cores are clearly observed and are surrounded by white circles corresponding to the protein shells. 

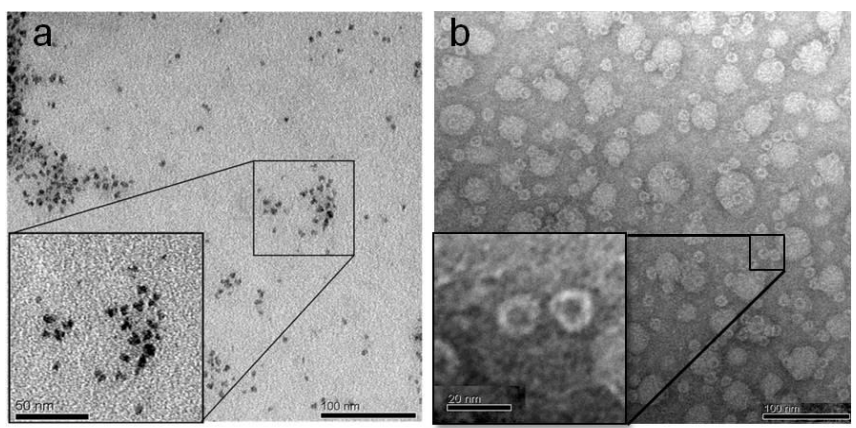

Fig. 1 Typical TEM images of a) non-stained and b) stained magnetoferritin samples. The small white circles correspond to the organic shells and the black dots correspond to the iron oxide material. The bigger white spots are due to inhomogeneous staining.

A particle size distribution was obtained from images taken in non-stained samples by measuring the longest side of each of the particles. Irregular shapes are commonly obtained for iron oxide particles grown inside apoferritin, due to the sensitivity of the nucleation and growth processes to environmental conditions.[15] These effects might be enhanced due to the presence of multiple oxidation and nucleation centres located in the inner protein cavity causing an unequal filling of the apoferritin cavity and giving uneven particles.[15, 28] The size distribution could be fitted with a Gaussian function giving an average diameter for the iron oxide cores of $5.7 \pm 1.6 \mathrm{~nm}$ (Fig. 2).

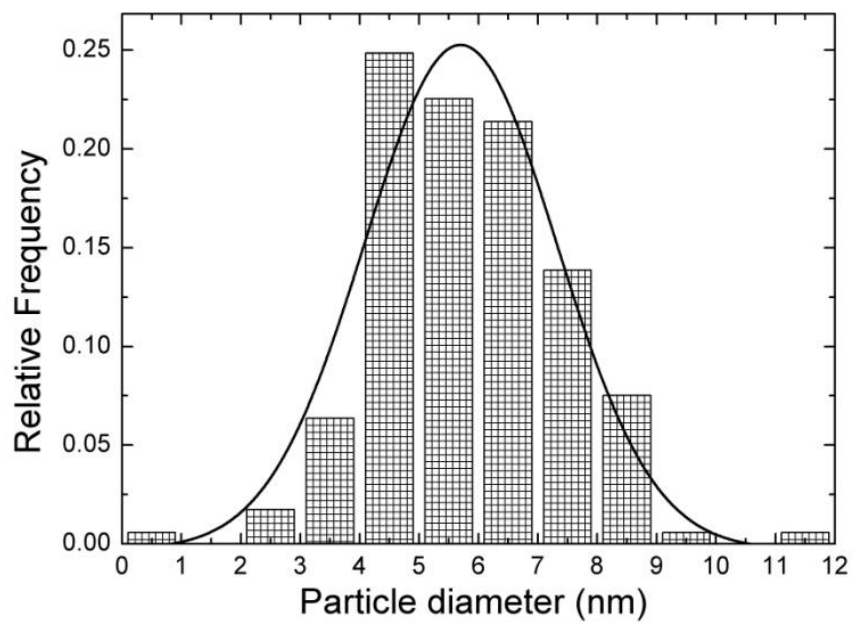

Fig. 2 The size distribution obtained from the analysis of TEM images was fitted using a Gaussian function giving an average diameter for the magnetoferritin cores of $5.7 \pm 1.6 \mathrm{~nm}$.

\subsection{Energy-dispersive $X$-ray spectroscopy $(E D X)$}


Two different micrometric areas were analyzed by EDX giving an average percentage of iron of 9.4 over the whole powder sample (SI Fig. 1 and SI Table I) that is equivalent to 958 iron atoms per protein molecule.

\subsection{Bulk magnetic properties: magnetization and ac susceptibility}

Magnetization isotherms measured at different temperatures above $40 \mathrm{~K}$ are shown in Fig. 3. In this temperature range, the magnetization is fully reversible, i.e. it does not show hysteresis, as expected for the equilibrium response of a superparamagnet. The high field magnetization is observed to increase with decreasing $T$, as shown in the inset of Fig. 3. This effect corresponds to the gradual decrease of the saturation magnetization as $T$ approaches the Curie temperature. The upturn observed below approximately $20 \mathrm{~K}$, which has also been observed in other samples of maghemite nanoparticles, has been ascribed to the gradual polarization of disordered spins located either at the outer surfaces or at the interfaces between different nanocrystals of the magnetoferitin core, $[2,15]$ which are subject to a much stronger local magnetic anisotropy than spins from the inner core. Such effects lead also to a strong reduction of the magnetoferritin average magnetic moment with respect to those found for crystalline maghemite nanoparticles of the same size.[15] 


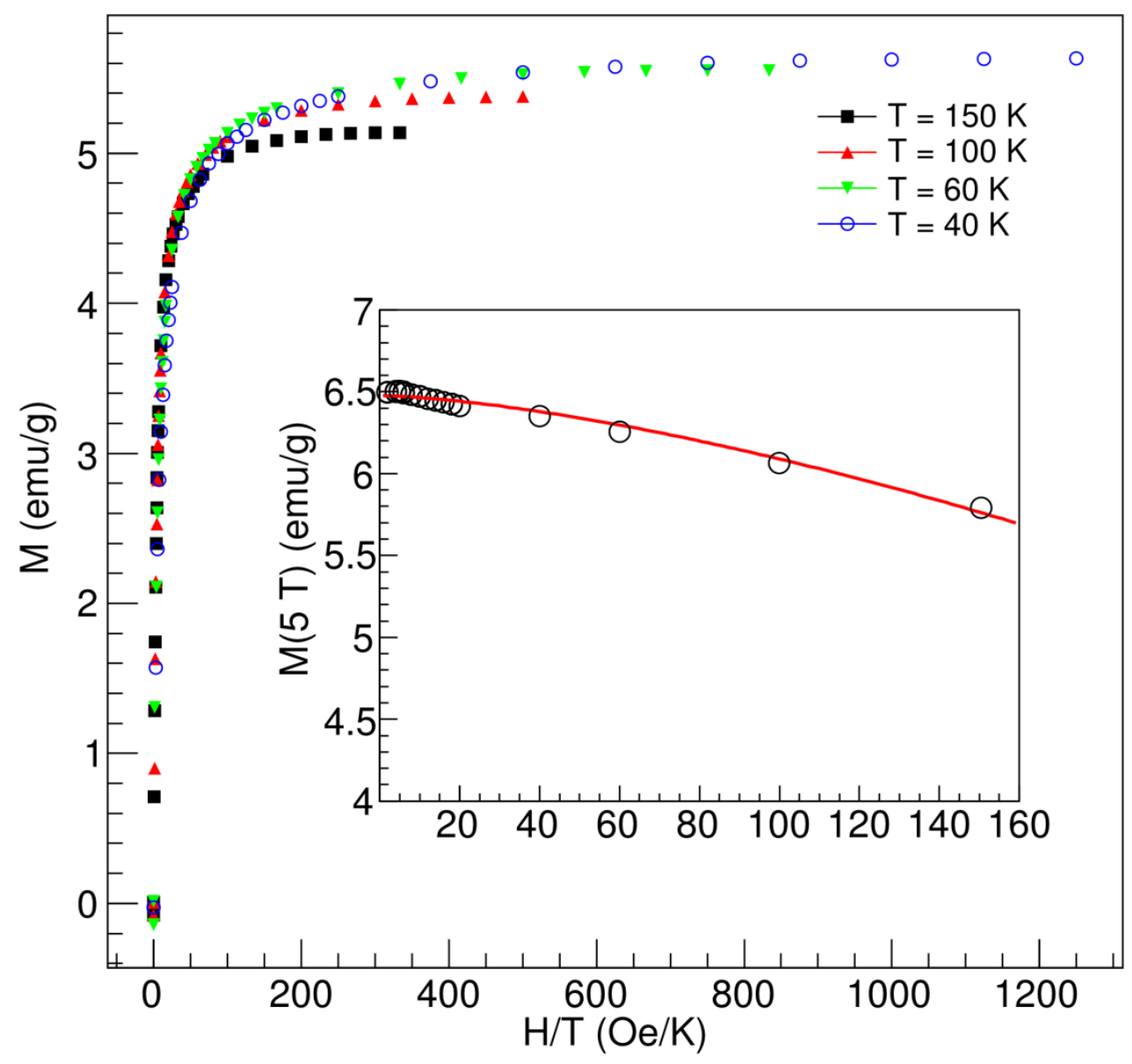

Fig. 3. Plot of the magnetization as function of the ratio $H / T$. Inset: Variation of the saturation magnetization with temperature. The solid line is a least-squares fit according to $M_{\mathrm{s}}(T)=M_{\mathrm{s}}(0)\left[1-\left(T / T_{\mathrm{c}}\right)^{3 / 2}\right]$, with $M_{\mathrm{s}}(0)=6.48 \pm 0.04 \mathrm{emu} / \mathrm{g}$ and $T_{\mathrm{c}}=650$ $\pm 60 \mathrm{~K}$.

The product $\chi T$, where $\chi$ is the dc susceptibility measured under a magnetic field of $100 \mathrm{Oe}$, is shown in Fig. 4. The slight decrease observed above $200 \mathrm{~K}$ is associated with the effect, just mentioned, that thermal fluctuations have on $M_{\mathrm{s}}$, which reduces the magnetic moment $\mu(T)$ of each nanoparticle and, therefore, its contribution to the magnetic susceptibility (it scales with $\mu^{2}(T)$ ). This effect can be accounted for by renormalizing the susceptibility by the factor $\left[1-\left(T / T_{\mathrm{c}}\right)^{3 / 2}\right]^{2}$, obtained from the fit of the saturation magnetization (inset of Fig. 3). The magnetic susceptibility renormalized by this factor $\tilde{\chi}$ follows Curie-Weiss law down to approximately $40 \mathrm{~K}$, as shown in the inset of Fig. 4. The Weiss temperature $\theta \approx-69 \mathrm{~K}$ determined from this fit gives the energy scale of interparticle magnetic interactions. 


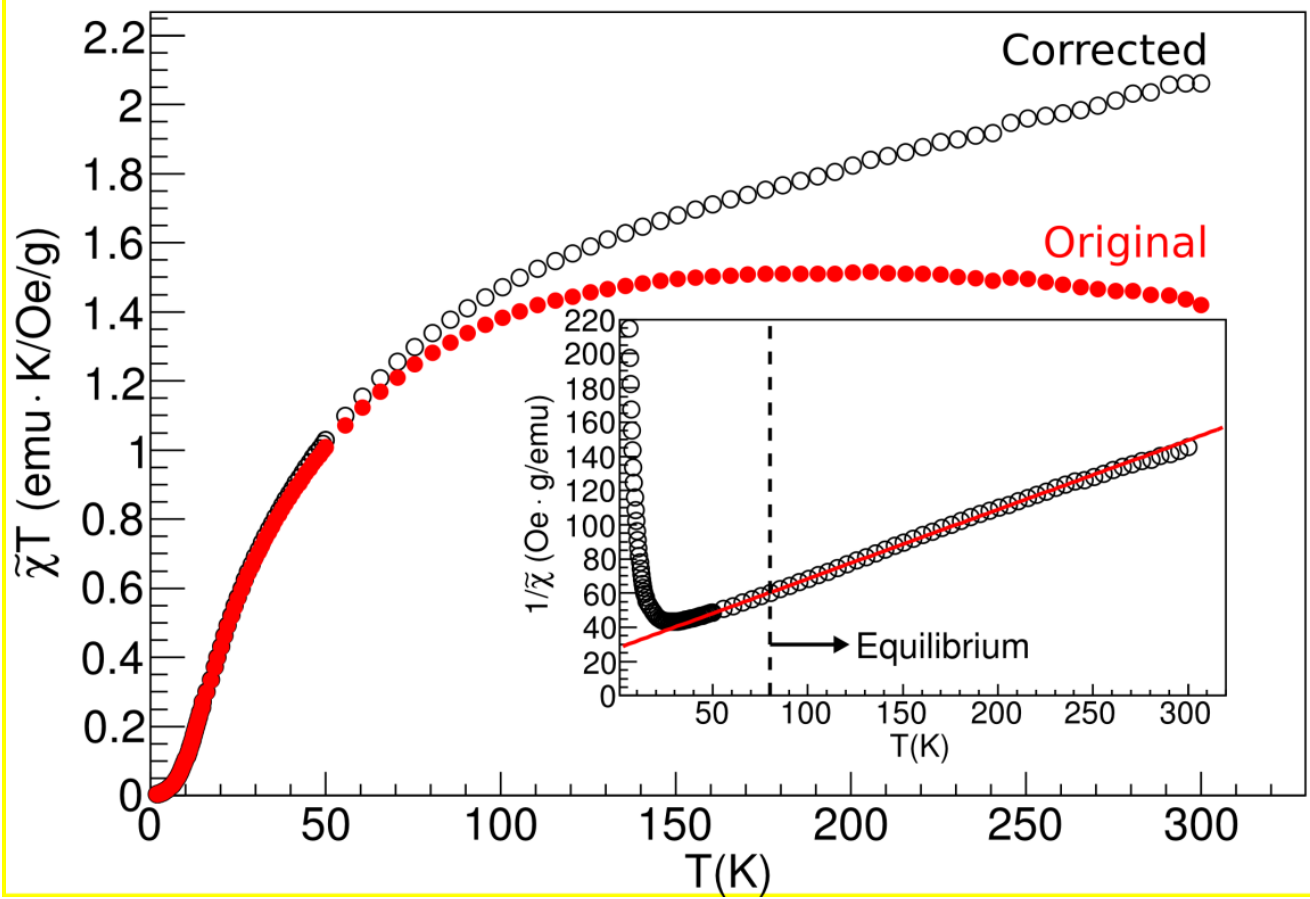

Fig. 4. $\chi T$ measurements of magnetoferritin in an applied constant magnetic field of 100 Oe. Solid dots: original $\chi T$ data. Open symbols: $\tilde{\chi} T$ data corrected by a factor [1$\left.\left(T / T_{\mathrm{c}}\right)^{3 / 2}\right]^{-2}$, which accounts for the reduction of the particles' magnetic moments by thermal fluctuations. Inset: Curie-Weiss fit of the reciprocal equilibrium susceptibility $1 / \tilde{\chi}$, measured above $40 \mathrm{~K}$.

A clear deviation of $1 / \tilde{\chi}$ from linearity (and a drop of $\tilde{\chi} T$ ) becomes noticeable below approximately $40 \mathrm{~K}$. It can be ascribed to the onset of non-equilibrium phenomena, i.e. to slow relaxation of the magnetization. The existence of magnetic irreversibility at low temperatures is evidenced also by the comparison, shown in Fig. 5 , of susceptibility data measured after either cooling the sample under zero magnetic field (ZFC) or in the presence of the measuring magnetic field (FC). The temperature $T_{i r r} \approx 80 \mathrm{~K}$ below which ZFC and FC curves begin to deviate from each other can be associated with the magnetic freezing of the largest particles, while the ZFC maximum at $28 \mathrm{~K}$ reflects the superparamagnetic blocking temperature $\left(T_{B}\right)$ of particles with close to the average size $(5.7 \mathrm{~nm})$.[29] The numerical derivative of the difference between the ZFC and FC curves gives information on the distribution of anisotropy energy barriers.[30] The overall distribution can be approximated to a lognormal function, in agreement with what has been previously observed in other magnetoferritin samples.[2] 


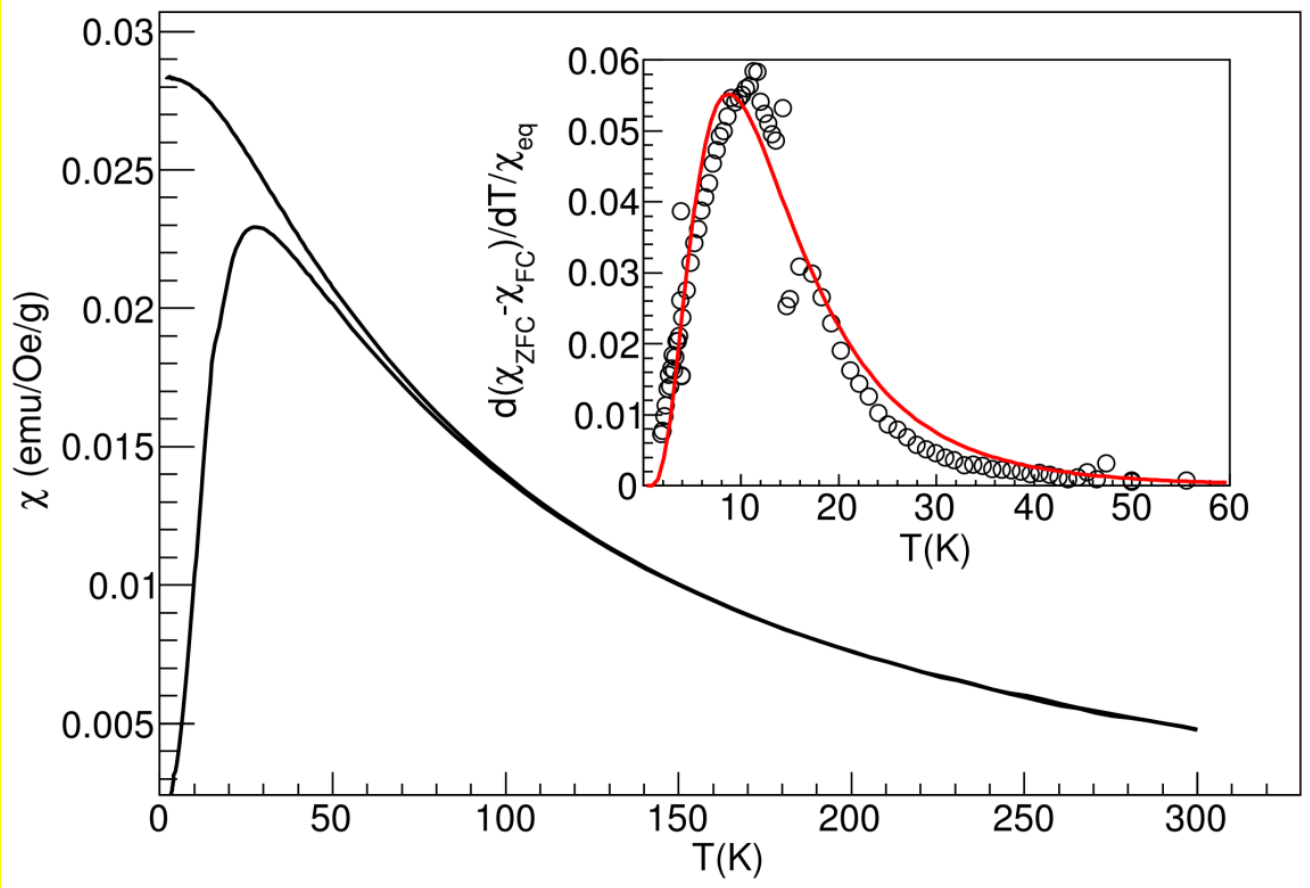

Fig. 5 Zero-field cooled (ZFC) and field-cooled (FC) magnetic susceptibilities of magnetoferritin measured under an applied magnetic field of 100 Oe. The inset shows the numerical first derivative of $\left(\chi_{\mathrm{ZFC}}-\chi_{\mathrm{FC}}\right)$, normalized by the equilibrium susceptibility $\chi_{\mathrm{eq}}=C /(T-\theta)$. This quantity is proportional to the size-induced distribution of anisotropy energy barriers. The line is a lognormal fit centred at $15 \pm$ $3.0 \mathrm{~K}$.

Below $T_{B}, M(H)$ curves begin to show hysteresis. Illustrative data are shown in Fig. 6. The coercive field $H_{c}$ and the remanent magnetization $M_{r}$ decrease with increasing temperature, nearly vanishing for $T>T_{B}$. The magnetisation quickly saturates above 4000 Oe affording a low $T$ saturation magnetisation $M_{s}=6.5 \mathrm{emu} / \mathrm{g}$. 


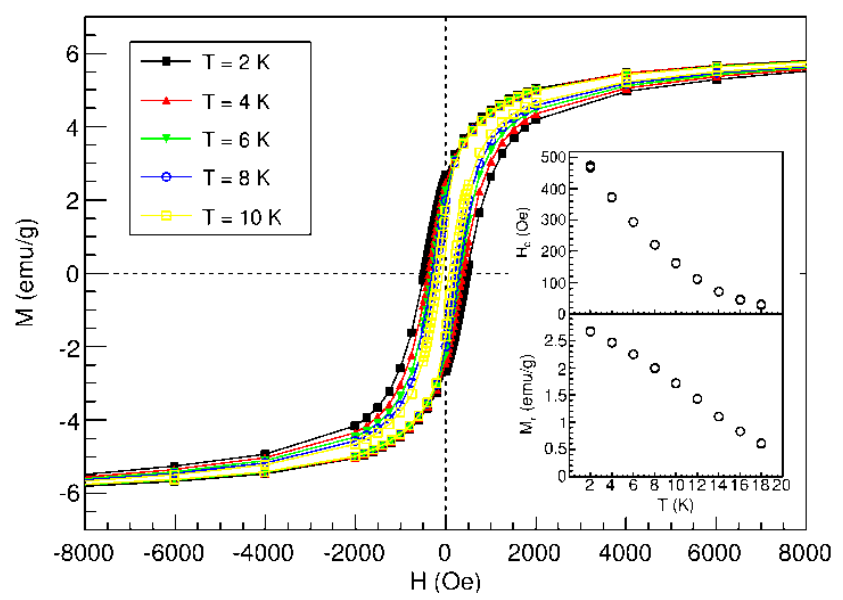

Fig. 3 Hysteresis loop measured at several temperatures. Inset: Dependence of the coercive field and remnant magnetization on temperature.

The dynamic properties were further studied by frequency dependent AC susceptibility measurements as a function of temperature (Fig. 7). Both the in-phase $\chi$ ' and the out-of-phase $\chi$ " susceptibility components show peaks at temperatures $T_{\max }\left(\chi^{\prime}\right)$ and $T_{\max }\left(\chi^{\prime \prime}\right)$ that shift to higher temperatures as frequency increases. Above $60 \mathrm{~K} \chi$ ' follows approximately a Curie's law and $\chi$ ” tends to zero. This means that the magnetic moments are in a superparamagnetic regime. Below $60 \mathrm{~K}$ both $\chi^{\prime}$ and $\chi$ ” show maxima. In analogy with the ZFC curve, the deviation of $\chi$ ' from the Curie's law can be interpreted as the blocking of the largest particles, which first deviate from the equilibrium Curie-Weiss law, whereas the maximum in $\chi$ " vs $T$ can be approximately ascribed to the blocking of the magnetisation of particles with average volume. We stress that, in general, the temperature dependences of $\chi$ ' and $\chi$ " near the blocking temperature depend on the distribution of anisotropy energy barriers $(U)$ and, in turn, on the width of the volumes distribution function.[30, 31] Therefore, in general $U$ ' and $U$ " obtained from Arrhenius fits of these maxima (see below) do not represent the energy barriers of the particles with average volume. 


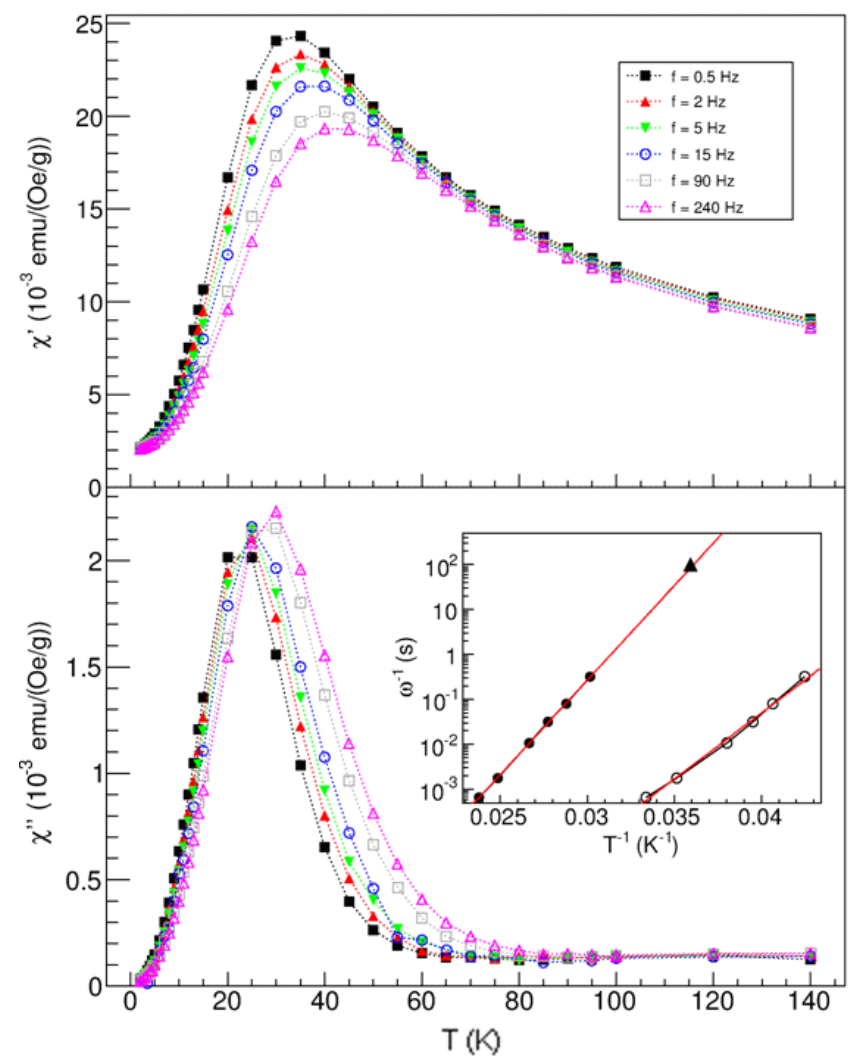

Fig. 4 Temperature dependence of the in phase (top panel) and the out of phase (bottom panel) components of the AC susceptibility measured at different frequencies of the oscillating 4.5 Oe magnetic field. Inset: Arrhenius plots of $T_{\max }\left(\chi^{\prime}\right)$ (solid circles) and $T_{\max }\left(\chi^{\prime \prime}\right)$ (open circles). The maximum temperature of the ZFC susceptibility is also shown (triangle). The solid lines are least square Arrhenius fits.

Both $T_{\max }\left(\chi^{\prime}\right)$ and $T_{\max }\left(\chi^{\prime \prime}\right)$ follow Néel-Arrhenius' law $1 / \omega=\tau_{0} \exp \left(U / k_{B} T_{\max }\right)$, characteristic of magnetic moments flipping via a thermally activated mechanism, where $\tau_{0}$ is the pre-exponential factor and $k_{B}$ is the Boltzmann constant. For the inphase maxima, we find $U^{\prime} / k_{B}=970 \pm 10 \mathrm{~K}$ and $\tau_{0}=4.0 \times 10^{-13} \mathrm{~s}$. For a time scale of $100 \mathrm{~s}$, typical of magnetization experiments, this law extrapolates to $T_{\max } \approx 29 \mathrm{~K}$, which agrees well with the blocking temperature estimated from the ZFC susceptibility (see Fig. 4 and the inset of Fig. 7). The analysis of the out of phase susceptibility gives $\mathrm{U}^{\prime \prime} / \mathrm{k}_{\mathrm{B}}=670 \pm 10 \mathrm{~K}$ and $\tau_{0}=1.26 \times 10^{-13} \mathrm{~s}$ which is in the range $10^{-13}-10^{-9}$ s found for similar systems, e.g. Pyrococcus Furiosus (PfFt) and Listeria Innocua (LiDps) behaving as independent superparamagnets. The first order magnetic anisotropy constant can be estimated as $K_{1} \approx U^{\prime \prime} / V=(5.6 \pm 0.1) \times 10^{4} \mathrm{~J} / \mathrm{m}^{3}$, where $V \approx$ $120 \mathrm{~nm}^{3}$ is the average particle volume according to the particle size distribution determined by TEM.[32] The estimated $K_{l}$ is one order of magnitude larger than the bulk $\gamma-\mathrm{Fe}_{2} \mathrm{O}_{3}$ value, i.e. $4.7 \times 10^{3} \mathrm{~J} / \mathrm{m}^{3}$ and this can be ascribed to two effects: i) the 
large surface to volume ratio of the nanoparticles grown into the protein cage gives rise to a large contribution of surface spins, with strong anisotropy $K_{s}$, to the overall anisotropy.[15] The surface anisotropy can be estimated from the relation: $K_{1}=K_{b u l k}+6 K_{s} / D$ where D is the average diameter of nanoparticles[33]. The result gives: $K_{s} \approx 7.3 \times 10^{-5} \mathrm{~J} / \mathrm{m}^{2}$ which is very close to the value estimated on similar magnetoferritin nanoparticles.[15] The positive $K_{s}$ value suggests that the surface spins are aligned preferentially along the surface of the nanoparticles rather than being oriented perpendicularly to their surface. ii) the inner core of magnetoferritin is very likely polycrystalline with each crystallite having its anisotropy axis along a different direction. Therefore, the magnetoferritin anisotropy is the result of the combinations of the single domain anisotropies within the inner core of protein cage which lead to magnetic symmetry lower than spherical (e.g. uniaxial as discussed in section 4) and in particular lower than that of the bulk $\gamma-\mathrm{Fe}_{2} \mathrm{O}_{3}$. Because the exchange interaction between the intra-particle domains is stronger than the magnetic anisotropy and the inter-particle interactions, each protein can be treated as a single domain with an effective magnetic moment and magnetic anisotropy estimated as above.

\section{Spin dynamics studied by electron magnetic resonance.}

\subsection{Spectral lineshape considerations and temperature dependence of EMR} intensities, linewidths and resonance fields.

The temperature dependence of EMR spectra measured at X and Q bands for a powder samples are reported in Fig. 8. The room temperature $\mathrm{X}$ band spectrum consists of a superposition of narrow and symmetric line centred at $B_{0}=337 \mathrm{mT}(\mathrm{g} \sim$ 2) and an asymmetric and broad line, moreover, a small feature is found at $\mathrm{B}_{0} / 2$. It is noteworthy that this feature is not due to $\mathrm{Fe}^{\mathrm{III}}$ impurities which occurs at $\mathrm{g}=4.3$. As temperature decreases, the narrow line decreases in intensity without shifting in field position while the broad line resonance field shifts to lower magnetic fields and broadens down to $5 \mathrm{~K}$ (Fig. 9). The Q band spectra consist of a single and symmetric line centred at $1184 \mathrm{mT}(\mathrm{g} \sim 2)$. Similarly to the $\mathrm{X}$ band result, upon cooling, the resonance field shifts to lower values and the linewidth broadens (Fig. 9). Similar X band results have been previously reported and were the subject of studies that account for quantum mechanical interpretation of the observed magnetic transitions [32, 34]. The overall EMR linewidth results from the contributions of 
homogeneous and inhomogeneous broadenings. The former is due to relaxation effects which are parameterized with a damping term in the Landau-Lifshitz-Gilbert (LLG) equation. The latter contains the contributions of the random distribution of anisotropy axes and distributions of magnetic anisotropy and magnetic moments due to the particle size distribution.

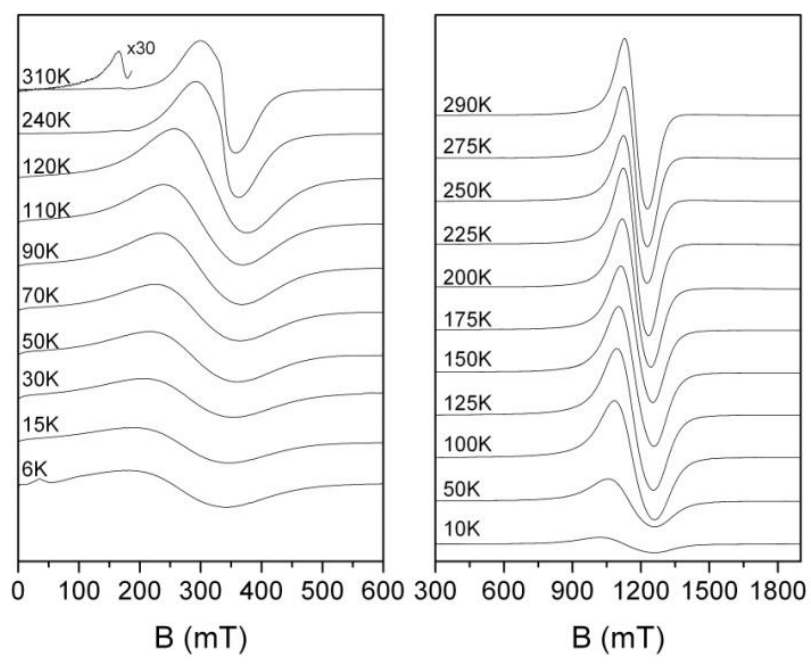

Fig. 5 X (left panel) and Q band (right panel) spectra for powder sample measured at different temperatures. The low field feature in the $\mathrm{X}$ band spectrum on the bottom is due to background paramagnetic impurities.
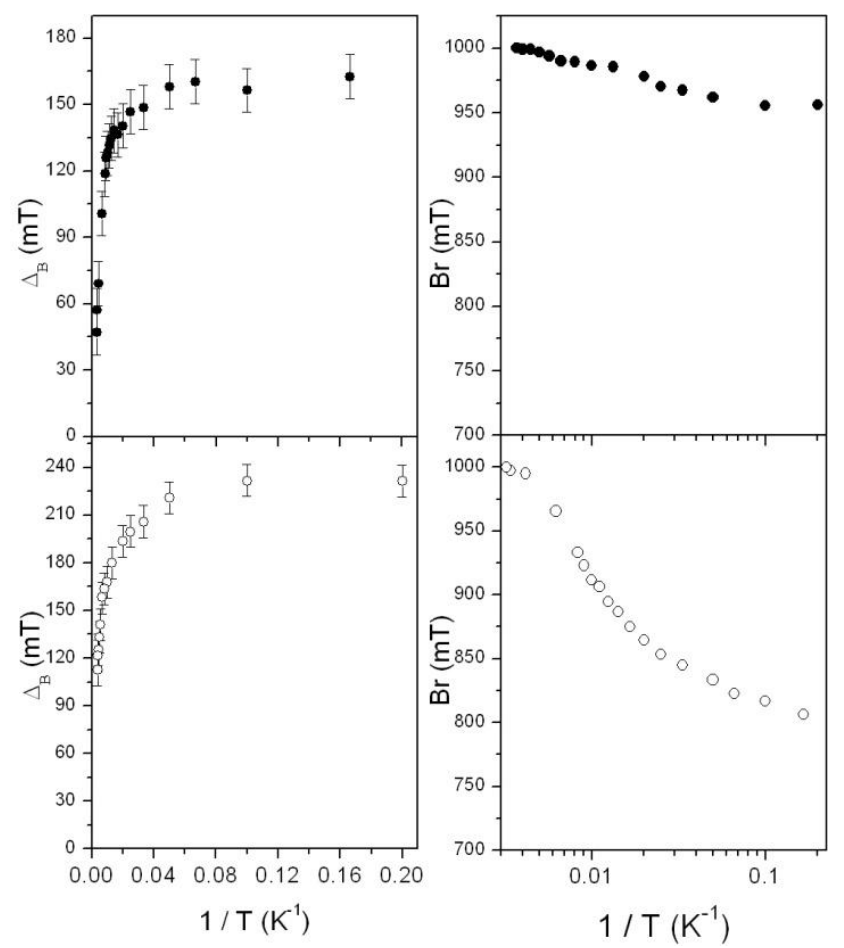

Fig. 6 Temperature dependence of the peak-to-peak linewidth (left panel) and resonance field (right panel) for X (top panel) and Q (bottom panel) band spectra. 
The double integrated area of the EMR spectra contains information on the out of phase component of the susceptibility at the employed frequency, static magnetic field and temperature. The temperature dependence at $\mathrm{X}$ and $\mathrm{Q}$ band reported in Fig. 10 shows a broad maximum at $110 \pm 5 \mathrm{~K}$ and $130 \pm 10 \mathrm{~K}$ respectively. In Fig. 10a we observe that the Q-band intensity maximum clearly shifts towards higher temperatures with respect to that observed at X-band frequencies. In Fig. 10b we report a qualitative comparison between the $\mathrm{AC}$ results of the Arrhenius plot extracted from Fig. 7 and those measured by EMR. These maxima are due to the blocking of the magnetisation, in analogy with the same phenomenon observed in the linear magnetic susceptibility, although in the case of EMR the frequency of the microwave magnetic field and the intensity of the static magnetic field are very different. One expects that, under the same experimental conditions, the much higher frequencies characteristic of EMR must lead to higher blocking temperatures, as it is indeed observed. However, a quantitative comparison between the two sets of data is complicated by the effect that the static magnetic field applied in EMR experiments might have on the activation energies for spin reversal. Still, Fig. 10b provides valuable information on how the spin dynamics depends on temperature over an unusually broad range.
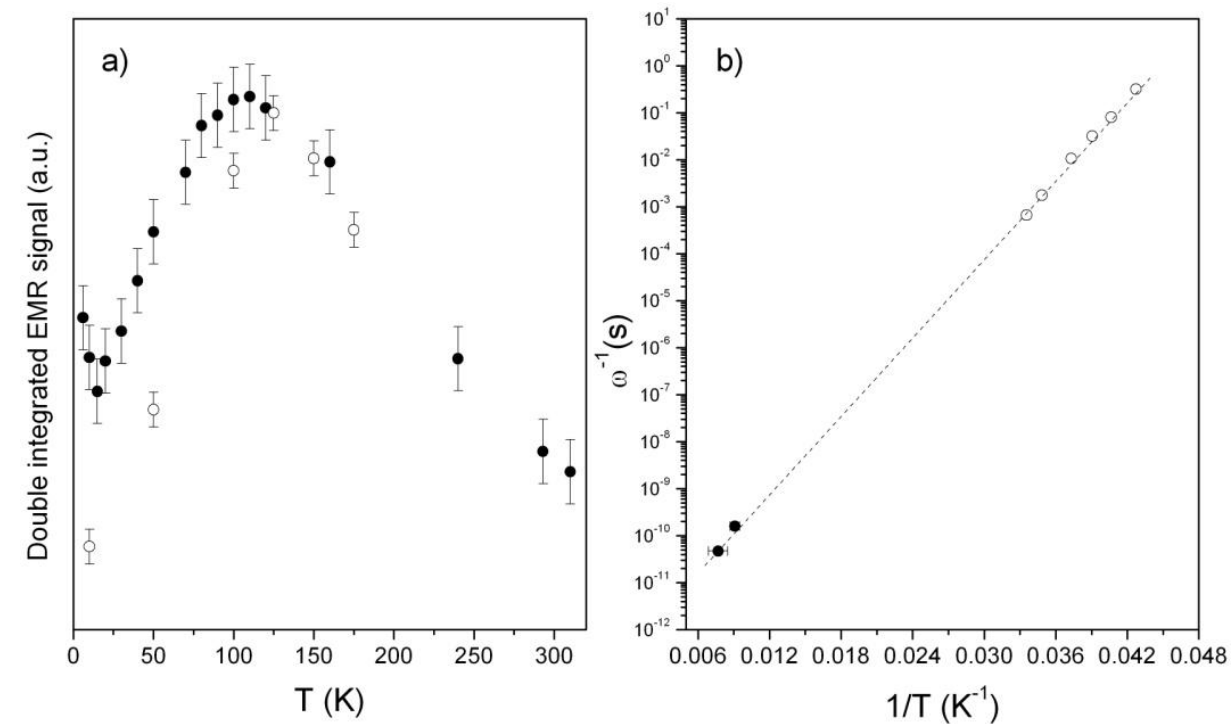

Fig. 7 a) temperature dependence of double integrated EMR signal for X-band (9.7 $\mathrm{GHz}$ ) and Q-band (34 GHz) spectra of powder sample. b) Arrhenius plot of $T_{\max }(\chi ")$ data as measured from SQUID (empty circles) and EMR (filled circles). The dashed line is a least-squares linear fit of data determined from SQUID measurements. 


\subsection{Frequency dependence of EMR spectra}

Multifrequency spectra on magnetoferritin powder sample at room temperature are shown in Fig. 11. The overall spectral lineshape is more structured at low frequencies and gradually evolves towards a more symmetric Gaussian lineshape at higher frequencies. The Gaussian lineshape of the high frequency EMR spectra shows that distribution of anisotropy parameters dominate the linewidth broadening. The spectral intensity of the narrow component at $\mathrm{g} \approx 2$ is clearly visible only at $\mathrm{S}$ and $\mathrm{X}$ bands where it accounts for $8 \%$ and $3 \%$ of the total absorption, respectively. This behaviour has already been observed on Fe-based nanoparticles formed in borate and silica glasses $[35,36]$ as well as in maghemite nanoparticles synthesized in biological moulds.[24, 35, 36]

The frequency dependence of $B_{r}$ and $\Delta_{B}$ is shown in Fig. 12. The resonance field scales linearly with frequency as follows from the magnetic resonance condition ( $\left.h v=g \mu_{B} B\right)$. The linear fit of the data provides a $g$ value of $1.99 \pm 0.02$. More interestingly, the peak-to-peak linewidth does not linearly increase with microwave frequency but saturates above $30 \mathrm{GHz}$. A similar saturation of the linewidth has been reported by Antoniak et al. [37] and suggested to indicate deviations from Gilbert damping. This increase of the linewidth at higher frequencies can be explained by the shortening of experimental time scale window which allows for the observation of faster relaxing transitions both within identical and their distribution. 


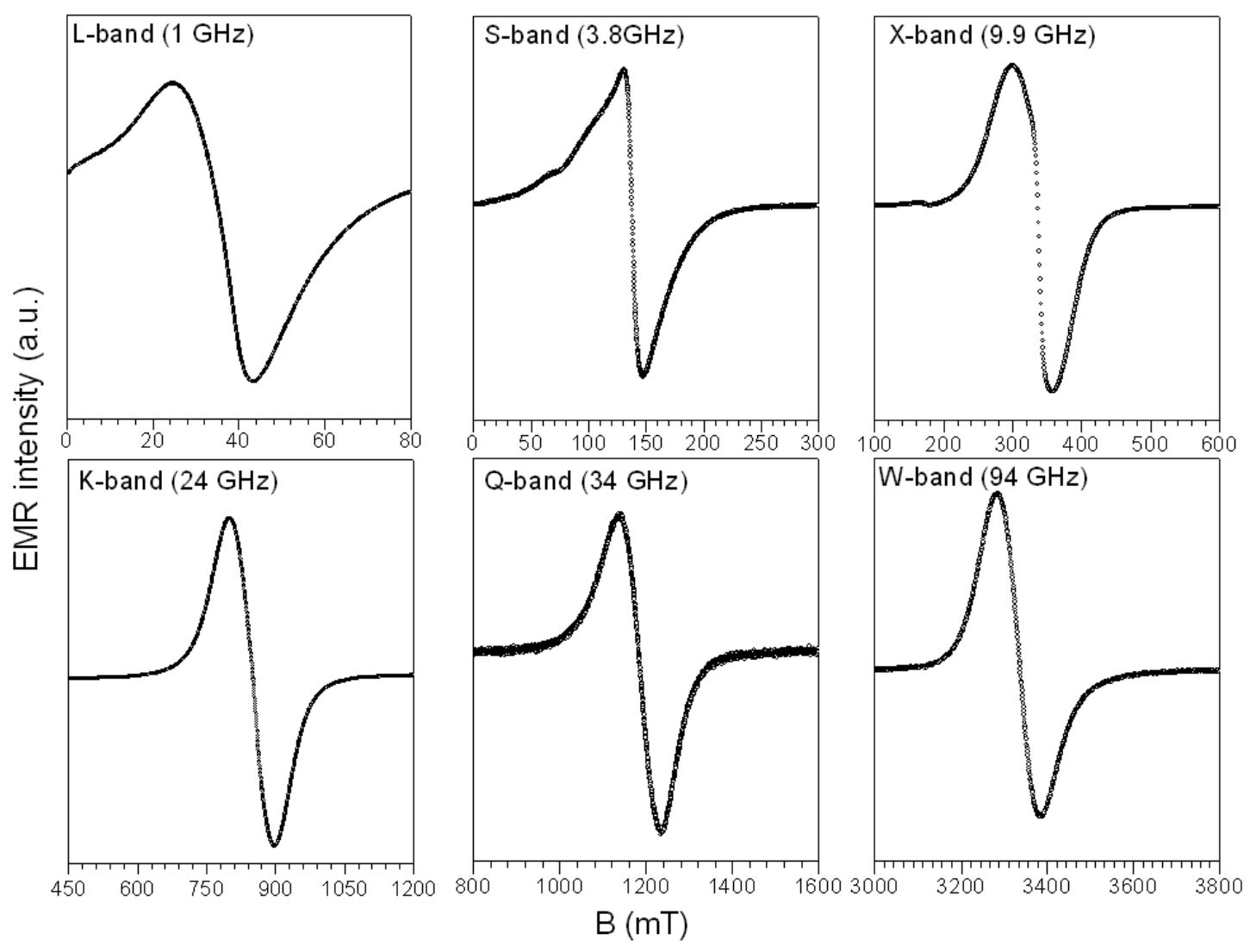

Fig. 8 Multifrequency EMR spectra for powder magnetoferritin at room temperature. 

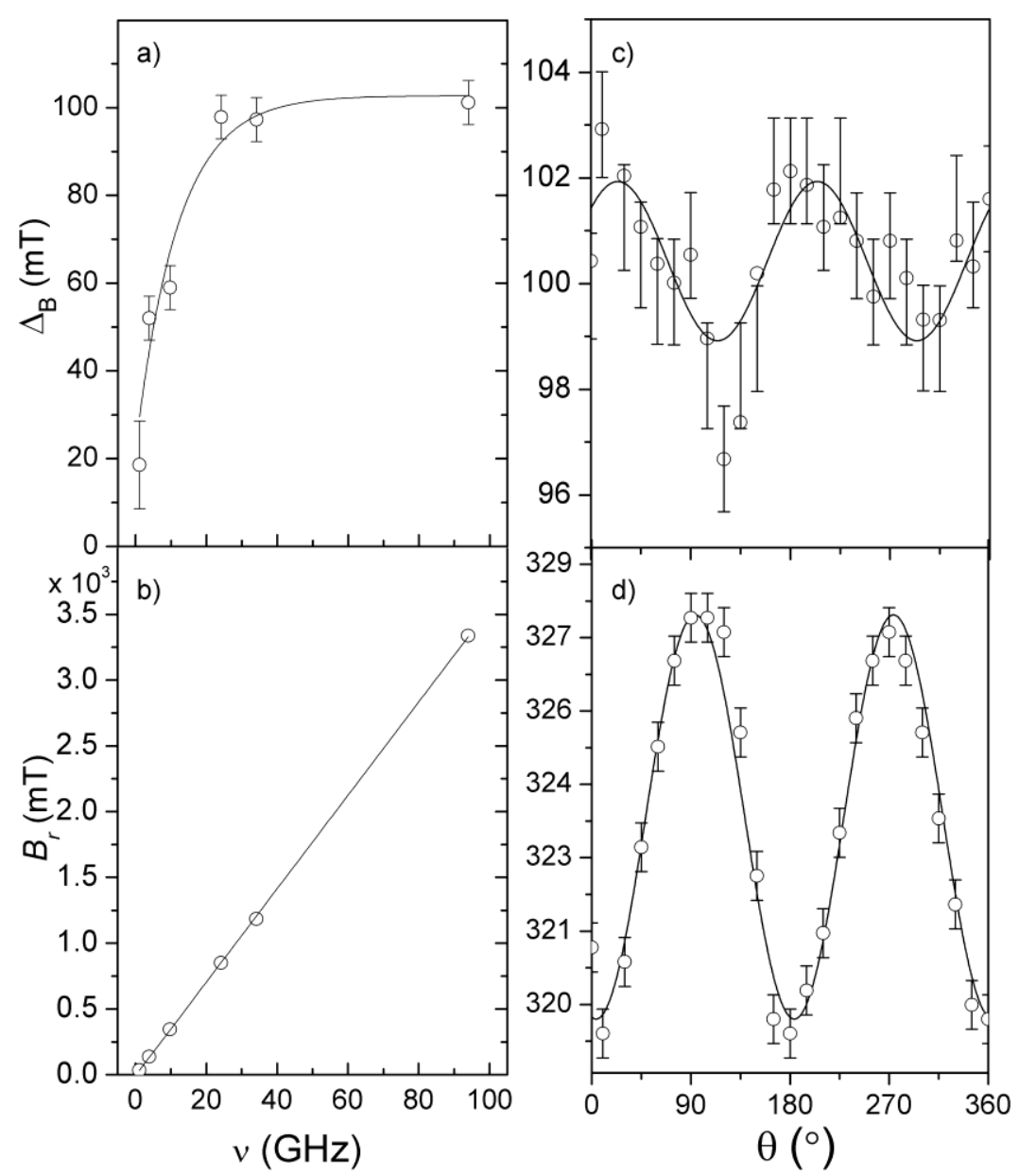

Fig. 9 Frequency dependence of the peak-to-peak linewidth (a) and resonance field (b) at room temperature for powder sample. Angle dependent peak-to-peak linewidth (c) and resonance field (d) at X band and $120 \mathrm{~K}$ after leaving the sample in an applied field of $1.5 \mathrm{~T}$ at $300 \mathrm{~K}$ for 10 minutes. The solid lines are the fittings (see text) while the dashed line is a guide for eye.

\subsection{Angle dependence of EMR spectra of frozen solution}

Angle dependent measurements on a frozen solution magnetic nanoparticles with aligned anisotropy axes permit the determination of the symmetry and anisotropy field $B_{a}$. The magnetic moments orientations result from a competition between the magnetic energy, which tends to align the magnetic moments along the external magnetic field, the anisotropy energy, which binds the magnetic moment to the easy axis, and the thermal energy, which tends to disorient both the magnetic moments and the anisotropy axes. In order to determine the symmetry and anisotropy field of the system under study, all particle anisotropy axes must be aligned along the same direction. This can achieved, with a good level of approximation, by freezing a solution of suspended nanoparticles after having applied a strong magnetic field. The 
nanoparticles, free to rotate in a suspended liquid solution, orient their magnetic moments and anisotropy axes along the direction of the applied field. The degree of alignment depends on the strength of the magnetic field and can be estimated by performing a field freezing (FF) experiment (Figure 13): EMR spectra are recorded at $\theta=0^{\circ}$ and $90^{\circ}$ with respect to the FF direction after having cooled the sample to 120 $\mathrm{K}$ (that is well below the glycerine freezing temperature and where the contribution of the narrow line at $\mathrm{g} \approx 2$ is negligible) under a given applied field. This procedure is then repeated for different field strengths. As an example, Fig. 13 shows the effect of a $1 \mathrm{~T}$ magnetic field on EMR spectra measured at $0^{\circ}$ and $90^{\circ}$ with respect to the FF direction, and compares it to spectra measured after freezing the solution at zero field. As expected, no angular dependence was observed for measurements performed after cooling in zero field because of the random distribution of anisotropy axes, whereas a shift towards higher and lower fields is found for spectra measured at $90^{\circ}$ and $0^{\circ}$, respectively when the sample is frozen in the presence of a magnetic field. The difference between the resonance fields at $90^{\circ}$ and $0^{\circ}$ is plotted as function of the $\mathrm{FF}$ strength in the right-hand panel of Fig. 13.
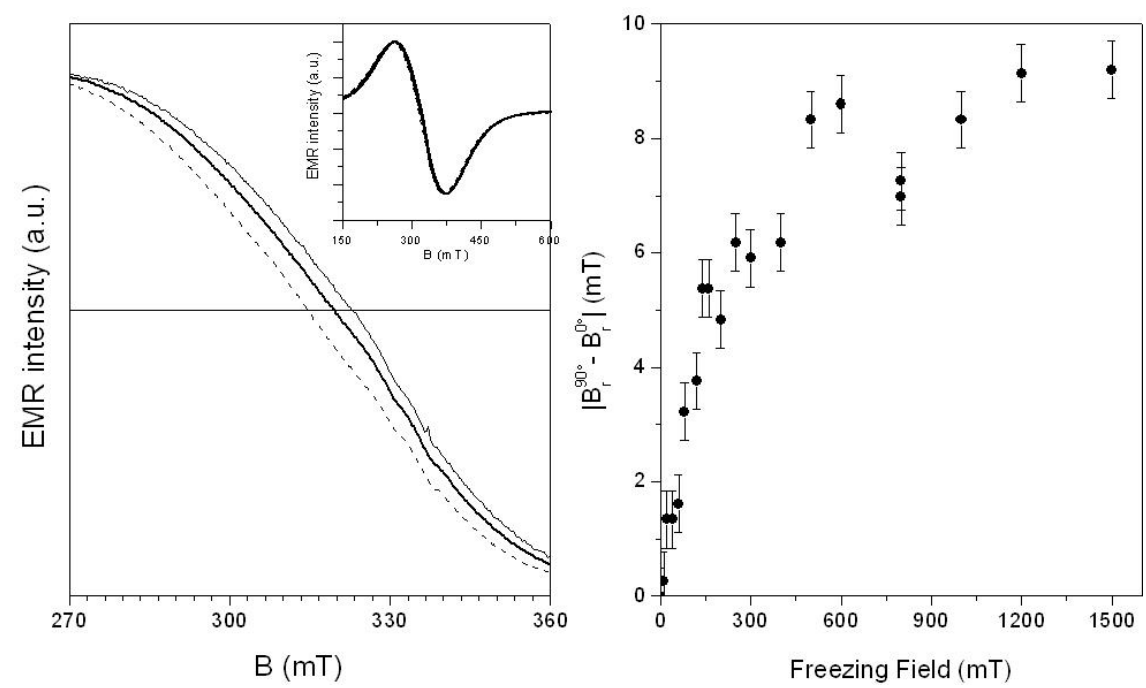

Fig. 10 Left panel: X-band EMR spectra measured, at $120 \mathrm{~K}$, on a frozen solution of magnetoferritin: thick line, data measured after cooling the sample at zero field; dashed and thin solid lines, data measured after freezing the solution under a magnetic field of $1 \mathrm{~T}$ for, respectively $\theta=0$ and 90 degrees. The inset shows the complete spectra. Right panel: difference between the resonance fields measured at 0 and 90 degrees for varying strengths of the magnetic field applied in the cooling down process.

In our EMR set-up, the external magnetic field is transversal to the oscillating magnetic field of the electromagnetic radiation (perpendicular mode). In this 
configuration, at zero degree the magnetic moment of magnetoferritin is saturated along the direction of the external field and the resonance field is minimised due to the additional contribution of the internal nanoparticle field. By rotating the EMR tube, the magnetisation of magnetoferritin is turned away from the FF direction and the resonance field shifts to higher fields, showing a maximum at $90^{\circ}$ before it goes again to zero at $180^{\circ}$. The results of these experiments are shown in Fig. 12, which shows two peaks for a full rotation of the sample giving evidence for a predominantly uniaxial magnetic anisotropy, rather than cubic as it would be expected for bulk $\gamma$ $\mathrm{Fe}_{2} \mathrm{O}_{3}$. A cubic symmetry would have resulted in four peaks for a similar experiment.[25] The fit of the experimental data to a $\left(B_{r}^{90^{\circ}}-B_{r}^{0^{\circ}}\right) \sin ^{2}(\theta)$ function gives an estimation of the internal field due to the combination of anisotropy and demagnetisation factors: $B_{i}=8.3 \pm 0.3 \mathrm{mT}$. We also found a two-fold symmetry for the angle dependence of the peak-to-peak linewidth (Fig. 12) which follows a $\sin ^{2}(\theta)$ dependence. It has been speculated that the angular dependence of the linewidth is due to the particle size distribution and hence to a distribution of anisotropy fields[27] which is clearly present in our case.

\section{Conclusions}

We studied polycrystalline magnetoferritin with average diameter of $5.7 \mathrm{~nm}$. The combination of SQUID and EMR experiments gives access to studying the magnetic susceptibility and magnetization dynamics on an experimental timescale ranging from seconds to nanoseconds, which is important information for practical applications. Our results show that the magnetic anisotropy of magnetoferritin nanoparticles is much stronger than that of bulk maghemite. This finding can be interpreted as result of the internal disorder of nano-crystals grown into the protein cages which lower the particle symmetry and/or to the their large surface to volume ratio. Our data substantiate a predominantly uniaxial magnetic anisotropy, with a considerable internal magnetic field.

\section{Acknowledgements}


We acknowledge funding from the Marie Curie Intra European Fellowship PIEF-GA2009-253980 (F.M.), EPSRC National UK EPR Facility and Photon Science Institute of the University of Manchester, Spanish MICINN under grants MAT2009-13977C03-01, MAT2009-13977-C03-02 and from DGA, under grants Biología Estructural and MOLCHIP. AL thanks ARAID for financial support. RdM and MJ are indebted to MICINN and CSIC for receiving pre-doctoral FPU and JAE fellowships, respectively. We thank Dr Jonathan Hawkett for assistance during EMR measurements.

\section{References}

[1] M. Y. M. T. Klem and T. Douglas, Materials Today 8 (2005) 28.

[2] S. Gider, D. D. Awschalom, T. Douglas, S. Mann, and M. Chaparala, Science 268 (1995) 77.

[3] F. C. Meldrum, B. R. Heywood, and S. Mann, Science 257 (1992) 522.

[4] R. Tsukamoto, M. Muraoka, Y. Fukushige, H. Nakagawa, T. Kawaguchi, Y. Nakatsuji, and I. Yamashita, Bulletin of the Chemical Society of Japan 81 (2008) 1669.

[5] S. Mann and F. C. Meldrum, Adv. Mat. 3 (1991) 316.

[6] T. Ueno, M. Suzuki, T. Goto, T. Matsumoto, K. Nagayama, and Y. Watanabe, Ang. Chem. Int. Edit. 43 (2004) 2527.

[7] N. Galvez, P. Sanchez, J. M. Dominguez-Vera, A. Soriano-Portillo, M. Clemente-Leon, and E. Coronado, Journal of Materials Chemistry 16 (2006) 2757.

[8] N. Galvez, P. Sanchez, and J. M. Dominguez-Vera, Dalton Trans. (2005) 2492.

[9] M. Clemente-Leon, E. Coronado, A. Soriano-Portillo, N. Galvez, and J. M. Dominguez-Vera, Journal of Materials Chemistry 17 (2007) 49.

[10] M. Uchida, M. L. Flenniken, M. Allen, D. A. Willits, B. E. Crowley, S. Brumfield, A. F. Willis, L. Jackiw, M. Jutila, M. J. Young, and T. Douglas, J. Am. Chem. Soc. 128 (2006) 16626.

[11] R. De Miguel, M. J. Martinez-Perez, M. Martinez-Julvez, S. Fiddyment, A. L. Garcia-Ortin, C. Gomez-Moreno, F. Luis, and A. Lostao, Febs Journal 278 (2011) 155.

[12] S. Laurent, D. Forge, M. Port, A. Roch, C. Robic, L. V. Elst, and R. N. Muller, Chem. Rev. 108 (2008) 2064.

[13] J. W. M. Bulte, T. Douglas, S. Mann, R. B. Frankel, B. M. Moskowitz, R. A. Brooks, C. D. Baumgarner, J. Vymazal, M. P. Strub, and J. A. Frank, JmriJournal of Magnetic Resonance Imaging 4 (1994) 497.

[14] K. K. W. Wong, T. Douglas, S. Gider, D. D. Awschalom, and S. Mann, Chemistry of Materials 10 (1998) 279.

[15] M. J. Martinez-Perez, R. de Miguel, C. Carbonera, M. Martinez-Julvez, A. Lostao, C. Piquer, C. Gomez-Moreno, J. Bartolome, and F. Luis, Nanotechnology 21 (2010). 
[16] J. G. Zhu, X. C. Zhu, and Y. H. Tang, IEEE T. Magn. 44 (2008) 125.

[17] Y. A. Koksharov, S. P. Gubin, I. D. Kosobudsky, G. Y. Yurkov, D. A. Pankratov, L. A. Ponomarenko, M. G. Mikheev, M. Beltran, Y. Khodorkovsky, and A. M. Tishin, Phys. Rev. B 63 (2001).

[18] K. Nagata and A. Ishihara, J. Magn. Magn. Mater. 104 (1992) 1571.

[19] R. Berger, J. C. Bissey, J. Kliava, H. Daubric, and C. Estournes, J. Magn. Magn. Mater. 234 (2001) 535.

[20] F. Gazeau, V. Shilov, J. C. Bacri, E. Dubois, F. Gendron, R. Perzynski, Y. L. Raikher, and V. I. Stepanov, J. Magn. Magn. Mater. 202 (1999) 535.

[21] L. F. Gamarra, W. M. Pontuschka, J. B. Mamani, D. R. Cornejo, T. R. Oliveira, E. D. Vieira, A. J. Costa-Filho, and E. Amaro, Jr., J. Phys-Condens. Mat. 21 (2009).

[22] C. Antoniak, J. Lindner, and M. Farle, Europhysics Letters 70 (2005) 250.

[23] M. Respaud, M. Goiran, J. M. Broto, F. H. Yang, T. Ould-Ely, C. Amiens, and B. Chaudret, Phys. Rev. B 59 (1999) R3934.

[24] R. J. Usselman, M. Klem, M. Allen, E. D. Walter, K. Gilmore, T. Douglas, M. Young, Y. Idzerda, and D. J. Singel, J. Appl. Phys. 97 (2005).

[25] F. Gazeau, J. C. Bacri, F. Gendron, R. Perzynski, Y. L. Raikher, V. I. Stepanov, and E. Dubois, J. Magn. Magn. Mater. 186 (1998) 175.

[26] N. Noginova, F. Chen, T. Weaver, E. P. Giannelis, A. B. Bourlinos, and V. A. Atsarkin, J. Phys-Condens. Mat. 19 (2007).

[27] H. Y. Li, M. T. Klem, K. B. Sebby, D. J. Singel, M. Young, T. Douglas, and Y. U. Idzerda, J. Magn. Magn. Mater. 321 (2009) 175.

[28] D. M. Lawson, P. J. Artymiuk, S. J. Yewdall, J. M. A. Smith, J. C. Livingstone, A. Treffry, A. Luzzago, S. Levi, P. Arosio, G. Cesareni, C. D. Thomas, W. V. Shaw, and P. M. Harrison, Nature 349 (1991) 541.

[29] P. Southern, A. P. Robinson, O. I. Kasyutich, B. Warne, A. Bewick, and W. Schwarzacher, J. Phys-Condens. Mat. 19 (2007).

[30] F. Luis, J. M. Torres, L. M. Garcia, J. Bartolome, J. Stankiewicz, F. Petroff, F. Fettar, J. L. Maurice, and A. Vaures, Phys. Rev. B 65 (2002).

[31] F. Luis, E. del Barco, J. M. Hernandez, E. Remiro, J. Bartolome, and J. Tejada, Phys. Rev. B 59 (1999) 11837.

[32] M. Fittipaldi, C. Innocenti, P. Ceci, C. Sangregorio, L. Castelli, L. Sorace, and D. Gatteschi, Phys. Rev. B 83 (2011).

[33] S. P. Gubin ed., Magnetic Nanoparticles (Wiley-VCH, Weinheim, 2009).

[34] M. M. Noginov, N. Noginova, O. Amponsah, R. Bah, R. Rakhimov, and V. A. Atsarkin, J. Magn. Magn. Mater. 320 (2008) 2228.

[35] R. Berger, J. C. Bissey, J. Kliava, H. Daubric, and C. Estournes, J. Magn. Magn. Mater. 234 (2001) 535.

[36] J. Kliava and R. Berger, J. Magn. Magn. Mater. 205 (1999) 328.

[37] C. Antoniak, J. Lindner, V. Salgueirino-Maceira, and M. Farle, Physica Status Solidi a-Applications and Materials Science 203 (2006) 2968. 